\title{
DESIGNING CONTEXT-AWARE INTERACTION: An Action Research Study
}

\author{
Carl Magnus Olsson \\ Ola Henfridsson \\ Viktoria Institute \\ Göteborg, Sweden
}

\begin{abstract}
Context-aware computing is an important research theme of ubiquitous computing. One of the most debated issues regarding context-aware applications is the extent to which such applications can capture the complexity of social context. This debate has been fueled by the fact that many of the documented context-aware applications convey a relatively simplistic view of context. The typical separation between human activity and context is problematic as context is not something simply in which interaction occurs.

This paper applies and evaluates the plausibility of Dourish's (2001a, $2001 b$, 2004) interactional context view for designing context-aware applications that transcend the mainstream design agenda in context-aware computing. On the basis of a canonical action research study, we develop and test design principles for context-aware applications that convey an interactional view of context. Recognizing the car not only as an excellent example of a ubiquitous information environment, but also as an interesting and complex use setting already commonplace in the modern community, we have implemented and assessed a car infotainment application to provide us with a firm illustration of what such a context-aware application might be.
\end{abstract}

Keywords Action research, design-oriented research, context-aware computing, interactional view of context, ubiquitous information environments

\section{INTRODUCTION}

Over the years, a key research theme in ubiquitous computing has been contextaware computing. This research theme has been directed at exploring applications with 
the capacity to support users by aligning implicit human activity with computing services (Abowd and Mynatt 2000). Embedded in dedicated computing environments, such capacity relies on models of user environments that can be dynamically applied, developed, and modified as a response to changing circumstances (Lyytinen and Yoo 2002a).

Given its centrality to context-aware computing, the notion of context has been much debated (see Abowd and Mynatt 2000; Dourish 2004; Schmidt et al. 1999). One pressing issue is the extent to which context-aware applications can capture the complexity of social context. The majority of documented context-aware applications only use identity and location in their attempts to capture user environment changes (Abowd and Mynatt 2000). As an example, the seminal Active Badge system (Want et al. 1992) uses identity and location for triggering automatic doors, automatic telephone forwarding, and customized computer displays (Weiser 1991). Even though evaluations of the Active Badge system document promising results, it has been argued that more complex social situations would require context-aware applications that draw on notions of context that transcend attempts to approximate context with location (see Schmidt et al. 1999).

The scarcity of contextual cues when modeling human interaction may risk reducing the usefulness of context-aware applications. Even though many problems can be traced to technical issues including wireless networking, processing capability, storage capacity, and displays (Want et al. 2002), the omission of social aspects of contextawareness is a considerable problem (Dourish 2001a, 2001b). In fact, applicationcentered context-aware computing research has typically assumed context to be a form of information that is delineable, stable, and separable from activity (Dourish 2004). In particular, the separation between human activity and context is problematic since context is "not something merely 'in which' interaction occurs" (Giddens 1984, p. 71). Addressing this problem, Dourish (2004) suggests an interactional view where context is understood as something relational, dynamic, occasioned, and arisen from human activity. This view not only embeds a richer notion of human interaction but also enables a balanced take on the socio-technical challenges associated with ubiquitous computing (see Lyytinen and Yoo 2002b).

Lately, design-oriented information systems research has been recognized as a valuable way of developing insights applicable to new classes of information systems (Hevner et al. 2004, Markus et al. 2002). In particular in combination with the action research method, it provides a plausible approach to assessing promising conceptual work in authentic milieus (Lindgren et al. 2004). In an attempt to investigate human interaction support provided by context-aware computing applications, we therefore conducted an action research study (Baskerville and Wood-Harper 1996) with the objective of developing and testing design principles for context-aware applications that convey an interactional view of context. Recognizing the car not only as an excellent example of ubiquitous computing but also as a complex use setting strongly integrated in our everyday lives (Walker et al. 2001), this effort was taken in the context of car infotainment systems.

In collaboration with a car manufacturer and an automotive systems integrator, we developed a prototype application called CABdriver Space for evaluating the plausibility of the interactional view of context (Dourish 2001a, 2001b, 2004) in an authentic 
setting. CABdriver Space is a hand-held context-aware computer game intended to stimulate in-car interaction for both enjoyment and safety purposes. In using a significant amount of car-related contextual information and involving both passengers and driver, the car infotainment context provides an interesting setting for exploring contextuality in context-aware computing. Our previous work on the CABdriver concept has provided methodological insights for innovative prototype evaluation (Olsson and Russo 2004) as well as a first exploration of the interactional impacts of CABdriver with regard to the feasibility and usefulness of the application concept in cars (Olsson 2004).

\section{DESIGNING INTERACTION THROUGH CONTEXT- AWARE APPLICATIONS}

The increasing miniaturization and connectivity of mobile devices have enabled a wider diffusion of mobile services in people's everyday life. Triggered by drivers such as convergence, mass-scale availability, and mobility, this diffusion opens up a wide array of new research issues associated with the development and use of ubiquitous computing services (Jessup and Robey 2002; Lyytinen and Yoo 2002b). As highlighted by Lyytinen and Yoo (2002a), one such issue is to integrate large-scale mobile services with models of specific user environments that can be dynamically applied, developed, and modified as a response to changing circumstances.

However, the current understanding of user environments in context-aware computing is relatively underdeveloped (Abowd and Mynatt 2000; Dourish 2001a, $2001 \mathrm{~b}$ ). Context is frequently assumed to be a form of information that is delineable, stable, and separable from activity (Dourish 2004). In this regard, most context-aware applications use identity and location as sole indicators of contextual changes (Abowd and Mynatt 2000; Schmidt et al 1999). Theoretical and methodological approaches are therefore needed that can cater for the assembly of social and technological elements characterizing ubiquitous computing environments (Lyytinen and Yoo 2002b).

While the relationship between technical and social elements in information systems indeed has been highlighted over the years (see, for example, Orlikowski 1992; Walsham 1996), design-oriented research targeting new classes of information systems typically benefit from domain-specific theoretical frameworks (Walls et al 1992). In the case of context-aware applications, Dourish (2004) presents such a theoretical framework which views context as an interactional problem. Drawing on phenomenology, this framework is an attempt to provide a basis for designing context-aware interaction. In doing this, it outlines four interrelated assumptions that can work as vehicles for informing such design (Dourish 2004, p. 22). The first assumption posits that "contextuality is a relational property that holds between objects or activities." In other words, context is not external to the activities unfolding in particular instances. Second, this means that "the scope of contextual features is definer dynamically." Context is shaped through ongoing action, rather than predefined irrespective of what takes place in time and space. Third, context is, therefore, "an occasioned property, relevant to particular settings, particular instances of action and particular parties to that action," and, fourth, it "arises from the activity." 
Dourish's work represents a promising research direction for the design of contextaware applications. However, much work is still needed to evaluate this direction in authentic settings. As highlighted by Abowd and Mynatt (2000), the delicate balance between prediction of the usefulness of novel technologies and observation of authentic use makes evaluation of ubiquitous computing research difficult. Indeed, the current wave of nomadic and ubiquitous computing environments challenges information systems researchers to adopt new research methodologies (Lyytinen and Yoo 2002b). In this vein, we designed an action research study for exploring the interactional view of context for designing a context-aware computer game intended to stimulate in-car interaction for both enjoyment and safety purposes.

\section{RESEARCH METHODOLOGY}

\subsection{Research Design}

The study reported in this paper was conducted as part of an action research project called "Mobile Services for In-Car User Value." The overall goal of this project was to explore different design challenges faced by car manufacturers that increasingly aim to support automotive commuters' everyday use of consumer electronics such as cell phones, mp3-players, PDAs, and smart phones. While one subproject targeted cell phone use and the issue of multi-contextuality (see Henfridsson and Lindgren 2005), the subproject presented here concerned PDAs and context-aware gaming.

Conducted between July 2002 and June 2004, the project was a lengthy collaborative study between the Viktoria Institute (research institute) and two firms operating in the automotive industry: Saab Automobile (car manufacturer) and Mecel (automotive systems integrator). Concurring with Robey and Markus' (1998) recommendation that practitioner sponsorship should be pursued to help overcome the commonly perceived rigor and relevance trade-off, the project was funded by the Swedish research agency VINNOVA together with the participating organizations.

Our action research followed the canonical action research method (Davison et al. 2004, Susman and Evered 1978) and thus corresponded to the five traditional phases: diagnosing, action planning, action taking, evaluating, and specifying learning. The diagnosing phase included collaborative (researchers and practitioners) workshop sessions and project meetings intended to identify core challenges in the car infotainment area. Documented through written notes and project minutes, these sessions and meetings focused on how the current automotive trend approaches interaction in cars (elaborated on in Olsson 2004) and how to design car infotainment that draws on the mobile setting of car traveling. This directed us to literature on context-aware computing and the notion of context. The diagnosing phase was followed by an action planning phase to specify actions that would allow us to test how an interactional view of context would affect design adapted to our specific setting. In collaboration with the practitioners, we developed three design principles that operationalized this theoretical framework into a design direction (see, for example, Walls et al 1992) intended to produce a meaningful context-aware application to assess from an automotive industry 
perspective. In the action taking phase, we developed a prototype, CABdriver Space, that embedded our design principles. In this regard, the prototype acted as research instrument for testing the plausibility of the design principles. Then, we recruited five families for evaluating the prototype over two months in their everyday life. The data collected and analyzed in the evaluating phase were later assessed jointly by researchers and practitioners for specifying learning with regard to both research and practice.

\subsection{Data Collection}

Concurring with the typical action research project, our data collection involved multiple data sources including project and workshop sessions, document review, lead user studies, open-ended questionnaires, and qualitative interviews (see Germonprez and Mathiassen 2004). Throughout the entire project, a total of 23 project meeting and workshop sessions were held and documented. These sessions were characterized by numerous discussion and decision points covering the infotainment concept, empirical studies, prototype development, and project deliverables.

As part of the two-month evaluation phase, semi-structured qualitative interviews (Patton 2002) were conducted with five families who had their cars modified for the evaluation of the CABdriver Space prototype. Because the prototype required a specific version of the Saab 9-3's infotainment platform, not commonly available to the public, the families could only be selected from a (fairly large) number of drivers from Saab Automobile. In order to avoid paying the price for making simplistic prototypes (Grudin 2001), we were forced to limit the selection of respondents in this way. However, it must be emphasized that none of the family members were associated with our project and were all known to provide precise and constructive feedback from previous in-house testing of other services.

Data was collected at two points of the evaluation period. Qualitative interviews were conducted half-way through the study. Consisting of both a joint family interview and individual interviews with each parent of the five families, these recorded and partly transcribed interview sessions lasted 150 to 210 minutes. During the family interviews, questions were formulated to encourage all family members to answer and they were specifically focused on tracing scenario descriptions of experiences from using the prototype. Questions ranged from appropriation questions (e.g., types of use and misuse, attitudes, conflict management, participation, changes in behavior, experimental use) to questions relating to their views on traveling by car with and without CABdriver, their expectations of what long-term use of CABdriver would mean to them, the future of the application concept, and their views on participating in the study. In covering similar questions, the individual interviews were conducted to trace individual differences between parents (because of their age, children were not interviewed individually).

The second data collection point consisted of an open-ended questionnaire designed on the basis of the interview round. It consisted of 27 questions corresponding to themes that emerged in analysis of the interview study. This questionnaire was answered in writing - one questionnaire per family-and followed up on how CABdriver had been appropriated, their perception on travel by car during this time, the 
long-term feasibility of the concept, possible future directions to consider implementing, and their feelings about being a part of the study. The questions were open-ended and again designed to stimulate participants to describe examples from the use to support their opinions.

\section{DESIGNING INTERACTION IN THE CAR INFOTAINMENT CONTEXT}

\subsection{Diagnosing}

On the basis of collaborative workshop sessions with the industry partners, we started our action research by exploring the emerging area of car infotainment and its possibilities and limitations vis-à-vis Saab Automobile's technical platforms and infotainment strategy. Given Saab Automobile's targeted market segment of "modern individualists who reject the mainstream," the action research group decided early on to explore a car infotainment direction that breaks with tradition. This was important to our automotive partner as they felt the automotive industry has long struggled with few significant innovations over the last decade - a result of the strict in-house prototyping and testing traditionally done. Saab Automobile strongly believed the industry as a whole had reduced external input that could have been used to foster new ideas to virtually none. The application was intended to utilize the information resources available from the in-car computing environment in order to benefit from the mobility aspects associated with car traveling. This breaks with the current trend for infotainment applications (including the rapidly growing introduction of gaming platforms such as Sony Playstation 2 ) in cars. These typically have no links to what goes on in or around the car. This intent also coincided well with our research agenda directed at investigating how to design meaningful interaction through context-aware applications.

In the attempt to explore the plausibility of a context-aware car infotainment concept, the action research group decided that testing it in an authentic setting would be a cumbersome but worthwhile direction. Given this ambition, we would need to develop a prototype that would work as a design test of Dourish's interactional context view.

\subsection{Action Planning}

We then set out to develop a set of design principles that would cater for Dourish's (2004) interactional context view. We focused our efforts on formulating this descriptive theory into a design direction intended to produce more meaningful contextaware applications (sce Walls et al 1992). Contrary to the context view held in mainstream context-aware computing literature, a core assumption in the interactional context view is that context arises from activity (Dourish 2004). With this in mind, we developed the following design principles: 
- The principle of relational context specifies that the context-aware application should support users' understanding of context through linking human activity (e.g., driver activity) with actions taken by the application. This principle corresponds to Dourish's (2004, p. 22) context assumption saying "contextuality is a relational property that holds between objects or activities."

- The principle of occasioned relevance specifies that the actions taken by contextaware applications should be triggered by occasions unfolding in the course of activity (e.g., driving) that are deemed relevant by the involved actors (e.g., driver). This principle corresponds to Dourish's (2004, p. 22) context assumption saying that "context is an occasioned property" rather than something that is stable.

- The principle of dynamic contextual scope specifies that the context-aware application should be able to determine dynamically the scope of contextual features that are relevant to process at a given occurrence of context-triggered action. This principle corresponds to Dourish's (2004, p. 22) context assumption saying that "the scope of contextual features is defined dynamically."

We realize that development of design principles from something which has a phenomenological foundation is somewhat problematic. From a phenomenological perspective, we want to avoid treating context in an instrumental way, but at the same time we need guidance when we make our design choices during development. Thus, at some point, any designer will have to take a stand and say "this is my intention" and try to implement in accordance with that. In our case, these intentions have been consciously guided by the design principles set out. The same applies to the design of our evaluation, as we also wanted to assess in what way these guiding principles were enacted at use time and what unintentional consequences the design brought. It is in the nature of design that, inevitably, choices have to be made, regardless if they may later be found to bring unintended consequences (hopefully together with what was intended).

\subsection{CABdriver Space: Action Taking}

CABdriver Space is a car infotainment prototype that is intended to provide entertainment experiences linked to travel by car. Saab Automobile supplied Saab 9-3 hardware and hardware modifications needed for CABdriver Space to be developed, as well as enabled the communication between hardware and infotainment system where this was not already available. Necessary adaptations to the operating system of the infotainment center, primarily the implementation of an improved IP stack, but also integration of the Bluetooth access point on the infotainment system, were handled by Mecel. Together, all three partners defined the data transfer protocol (using TCP/IP as the carrier) to be used when communicating from the infotainment system to a PDA running Pocket PC, on which the Viktoria Institute developed the game. Together, this platform communicated data from the car, including from car support systems (e.g., traction control system, electronic stability program, anti-brake locking), current speed, fuel consumption, GPS position, RDS-TMC (radio wave traffic messages), and tempera- 
ture to the PDA. The PDA in turn matched the position of the car with a geographical database to provide information such as gas stations, restaurants, parking spaces, speed limits, and road network.

In our desire to test Dourish's interactional context view in a design setting, we were instrumental in designing CABdriver Space so that it stimulated interaction between player and driver in a way that is adapted to the sensitive setting of a car. The player and her interaction with the driver are thus an important part of the prototype, which also further explains the name: Context-Aware Backseat Driver. CABdriver Space does not become meaningful (or indeed context-aware) unless the player and driver are able to associate the information affecting the game with the real world and use it to adapt their behavior accordingly. Screenshots and further explanations of CABdriver Space are provided in the Appendix.

Embedded in CABdriver Space are the three design principles we defined: relational context, occasioned relevance, and dynamic contextual scope. First, the game embeds the relational context principle by stimulating certain forms of interaction between passenger, driver, and application. The prototype is focused on object-activity, object-object, and activity-object relations, all striving to promote interaction adapted to the specific use setting. Examples of such design choices were $1: 1$ relations between turn signal and stress level (object-activity), momentary fuel consumption and the regeneration ability in the game (object-object), and excessive speeding and triggering of police mines (activity-object relation, greatly increasing the difficulty of the game). However, designing for the emergence of a specific meaning upon use is never an easy task. Given that context arises in action (Dourish 2004), context-aware applications that rely on this assumption have to come to terms with the fact that specific activity-activity relations are only enacted between humans during use and may not, at design time, be implemented in other ways than suggestions of certain behavior.

Second, the game embeds the occasioned relevance principle by coupling game events with occasions unfolding in the course of driving/traveling that are deemed relevant by the involved actors. For instance, CABdriver Space is designed to be significantly harder to play in the case of aggressive driving. This creates user incentives to influence the driver to drive more carefully. However, the learning is not unidirectional only (i.e., the passenger learning to understand the driver), but is also going in the opposite direction. In other words, the intent of the game is also to support the driver in linking her driving behavior to passenger responses. This bidirectional learning is intended to support the driver and passenger to jointly define the relevance of the context-triggered actions taken by the application. In order to enable such support, however, it is central that such triggers are in a satisfactory way coupled with occurrences in the course of driving.

Finally, the game embeds the dynamic contextual scope principle by assigning the actors the role of adapting the scope of contextual information used vis-à-vis the current situation. For instance, with CABdriver Space, the passenger has an important role in adapting her interaction with the driver to current driving conditions. Given that artifacts are unable to assign meaning to many complex driving situations, the passenger's ability to create accurate mental models - as well as to interpret the driver's mental model - becomes a key factor in order for the passenger to adapt the scope of contextual information to be shared with the driver. Using the driver workload 
parameter (in a Saab 9-3, the current driver workload depends on acceleration and braking, recent use of turn signal, electronic stability program activity, anti-spin control activity, and so on), CABdriver Space displays driver workload at all times as a small traffic light in the upper right corner of the screen. As illustrated in the Appendix, high driver workload makes the game more challenging to play, which in turn intends to make the player more focused on the game.

\subsection{Evaluating}

The CABdriver space prototype was evaluated over a two month period (November 2003 to January 2004). The evaluation included five cars and five Swedish families. At a meeting at Saab Automobile's head office in Trollhättan, Sweden, the families were introduced to the rationale behind our prototype design and evaluation strategy. We explained that they were expected to use CABdriver in their everyday driving as they themselves saw fit. In our desire for realistic evaluation conditions, Saab Automobile modified the hardware of the five cars involved, Mecel updated the infotainment center operating system, and each family was provided a PDA on which the Viktoria Institute had installed CABdriver Space.

The first design principle, relational context, appears to have had a significant impact on the participants. At design time, our desire was to inscribe certain behavior (safe and cautious driving through interaction between player and driver) into the relational aspects between activity-object, object-object, and object-activity. These appear to have largely played out as intended, creating activity-activity relations at use time, through the interaction our context-aware application was designed to stimulate.

The family appreciated feeling that they better understood each other as well as the car and surrounding through the context-aware application.... We prefer that something is dependant of the surroundings and the driving. You are influenced in a favorable way.... Initially, the missions themselves were most appreciated by the players, but later to be able to affect driving and navigation was most rewarding. For the driver, it was most liked because your driving could affect the difficulty of the game. I have become a wiser, more aware driver. (Jan, Family 2)

Nevertheless, not only the intended consequences have been observed. One unintended consequence comes from the duality that some design choices may bring with them. In our case, this was the relation we put between use of turn signal and driver workload. As we purposely designed the game to become harder when driver workload went up, participants soon figured out that if they did not use their turn signal (when judged safe), they did not transmit a higher driver workload to the game and therefore actively helped the player rather than make it harder for them (an issue further discussed later in the evaluation). 
As driver, you want to see how fast these inputs, like acceleration, affect regeneration [in the game]....You feel that you are a bit like God. (Thomas, Family 5)

Go easy on the gas, use turn signal selectively, [and] do not speed. (Family 5)

Related to the turn signal implementation issue, drivers and players clearly reported how interaction initiated by the player was not always adhered to strictly in the fashion the player desired. On some occasions, players demanded a particular response from the driver--a response not necessarily adapted to the traffic situation.

The player has asked the driver not to brake, for instance when driving in the city, which can become annoying for the driver after a while. You try not to brake, in order to avoid the nagging, which in turn creates dangerous traffic situations. (Per, Family 3)

Quit using the turn signal or the big monster will come! (Thomas, Family 5, about Fredrik, his frequent passenger)

Either you mess with him and use your turn signal extra, or you change lanes without using it. (Thomas, Family 5, describing his response to Fredrik)

This indicates that the contextual scope is dynamically defined (design principle three). The scope of which factors are relevant is quite different in one situation (e.g., stimulating smoother driving) while in another deemed less relevant to adapt to (e.g., it may be difficult to avoid hard braking in city driving). In other words, the scope has occasioned relevance (design principle two).

While we were not unaware that the particular scenario of smooth driving in city traffic may become an issue to the participants, we had hoped this would be settled by preemptive interaction from the driver, warning the player of what was ahead and explaining why this had to be so when she could not adapt a smoother style of driving. After all, during the three weeks the action research development team spent driving a Saab 9-3 to test the functionality and balance the application prior to the evaluation, we became aware of this possible issue, but after some training, we managed to negotiate rush-hour traffic without running into the problem discussed here. Although we managed to negotiate rush-hour traffic, the implementation may still incur a step that is too large to take for some drivers. In particular, this may be relevant for drivers that perceive much of the enjoyment in driving to lie in challenging conditions (Walker et al 2001), just as the father of Family 4 reflected on about his own driving. This is, therefore, an important design issue to resolve in order to avoid having the intended interaction lose its meaning as the difficulty level of the game becomes too high, thereby greatly affecting the ability of the application to support context-awareness.

Interaction has lessened [due to the difficulty level] and the children use it more like as a stand-alone game now [ignoring the option to interact] compared to when we started. The marble game on the PDA is a tough competitor. (Family 4) 
In a sense, even this loss of meaning, promoting competition from context-unaware applications, illustrates how important the specific relevance of a particular scope in a given situation is for an application to promote an interactional view on contextawareness during use.

\section{DISCUSSION}

Context-aware computing is one important research theme of ubiquitous computing (Abowd and Mynatt 2000). In this theme, the notion of context has frequently been debated (e.g., Dourish 2004, Schmidt et al. 1999). Commonly, this debate comes from the relatively simplistic view of context that many of the documented context-aware applications convey. In these, context tends to be treated as a form of information that is delineable, stable, and separable from activity, and thus tends to disassociate human activity and context (Dourish 2004). This becomes problematic as context is "not something merely 'in which' interaction occurs" (Giddens 1984, p. 71).

This paper addresses the notion of context by applying and evaluating the plausibility of Dourish's interactional context view for designing context-aware applications. On the basis of a canonical action research study, we have developed and tested design principles for context-aware applications that convey such an interactional view of context. This research agenda resonated well with that of our industrial partners and formed the common ground for the collaboration. The design principles developed were (1) relational context, (2) occasioned relevance, and (3) dynamic contextual scope.

In our two month evaluation in the everyday life of five Swedish families, we discovered that the CABdriver Space prototype-and our design principles embedded in it-was generally appreciated by our evaluators. For instance, the prototype's stimulation of interaction between driver, passengers, and application contributed to an increased contextual understanding of the driving situation on behalf of both drivers and passengers. By embracing the theoretical assumption that context arises from activity, the magnitude of meaningful interaction triggered by the prototype contributed to our respondents' more reflective and sound driving. This indicates the general plausibility of the interactional context view for informing the design of context-aware applications.

Our research also indicates that applications that draw on this context view can produce unintended consequences. For instance, the evaluation showed that our attempt to trigger adapted behavior to the driving situation by linking the driver workload metric (supplied by the car) to the game plan was ambiguous. Even though the turn signal (as one of the parameters in this metric) typically indicates a busy driver, the relation between an object (the turn signal) and activity (turning) does not always mean that the driver actually perceives a higher workload. In fact, our respondents described how they on some occasions deliberately neglected to use the turn signal as they did not want to transmit a high workload to the game. This shows how contextuality arises not only in the course of action, but also how it depends on the specific relationship between objects and activities (design principle one). Furthermore, this illustrates well how the scope of relevant contextual features is dynamically defined (design principle three): in one instance, very relevant (such as rush hour traffic), while in another (simple lane change on a lonely highway), it is perceived as less important (design principle two). 
On a general level, our research confirms Lyytinen and Yoo's (2002b) observation that it is increasingly difficult to distinguish between human and nonhuman actors in ubiquitous information environments. Context-aware applications perfectly exemplify the role of machine agency in human interaction. In fact, designing context-aware applications is largely about designing context-aware interaction, meaning that designers cannot oversee the contextual understanding needed for assessing the consequences of their design products. Given the scarcity of authentic evaluations in application-oriented context-aware computing research, our action research suggests a valuable way forward in handling the delicate balance between prediction of usefulness of novel technologies and observation of realistic use (see Abowd and Mynatt 2000). Drawing on a socially informed theoretical framework, it does so by offering a novel technology that is tested without the constraints and limitations of an experimental setting.

On a practical level, our action research contributed to Saab Automobile's dwelling with their car infotainment strategy and the state of innovation in the automotive industry as a whole. By participating in this project, Saab gained experience valuable for taking their attempts to break with the mainstream car infotainment agenda further. As the infotainment product manager was one of our key action research members, this learning is likely to have a strong organizational basis at Saab-useful for future releases of car infotainment concepts and services.

\section{CONCLUSION}

In this paper, we set out to evaluate the plausibility of an interactional view of context (Dourish 2001a, 2001b, 2004) by collaboratively developing a prototype application using an action research approach that is implemented through three design principles we defined: (1) relational context, (2) occasioned relevance, and (3) dynamic contextual scope. The contribution of this effort is to address the relative scarcity of contextual cues used when modeling and evaluating human interaction together with technology - an important issue within the rapidly growing theme of ubiquitous information environments in IS research. In particular, when combined with lab-like and other artificial evaluation settings, this scarcity may seriously affect the understanding and development of applications supporting context-awareness, a key area in ubiquitous computing (Abowd and Mynatt 2000).

Using the (hardware and software modified) Saab 9-3s of five families, our evaluation of CABdriver Space in everyday situations shows how the magnitude of meaningful interaction triggered by the prototype contributed to our respondents' more reflective driving. This confirms the plausibility of the interactional context view. Despite this, our results still call for more sophisticated theoretical frameworks that can capture the socio-technical complexity of designing context-aware interaction. We believe that the inherent machine agency component of context-aware applications needs more attention in addressing the notion of context as it emerges during use in ubiquitous information environments. 


\section{REFERENCES}

Abowd, G. D., and Mynatt, E. D. "Charting Past, Present, and Future Research in Ubiquitous Computing," ACM Transactions on Computer-Human Interaction (7:1), 2000, pp. 29-58.

Baskerville, R. L., and Wood-Harper, A. T. "A Critical Perspective on Action Research as a Method for Information Systems Research," Journal of Information Technology, 11, 1996, pp. 235-246.

Davison, R. M., Martinsons, M. G., and Kock, N. "Principles of Canonical Action Research," Information Systems Journal (14), 2004, pp. 65-86.

DeSanctis, G., and Scott Poole, M. "Capturing the Complexity in Advanced Technology Use: Adaptive Structuration Theory," Organization Science (5:2), 1994, pp. 121-147.

Dourish, P. "Seeking a Foundation for Context-Aware Computing," Human-Computer Interaction (16:2-4), 2001, pp. 229-241.

Dourish, P. "What We Talk About When We Talk About Context," Personal and Ubiquitous Computing (8), 2004, pp. 19-30.

Dourish, P. Where the Action Is: the Foundations of Embodied Interaction, Cambridge, MA: MIT Press, 2001b.

Giddens, A. The Constitution of Society-Outline of the Theory of Structuration, Cambridge, UK: Polity Press, Cambridge, 1984.

Germonprez, M., and Mathiassen, L. "The Role of Conventional Research Methods in Information Systems Research," in Information Systems Research: Relevant Theory and Informed Practice, B. Kaplan, D. P. Truex III, D. Wastell and A. T. Wood-Harper, and J. I. DeGross (Eds.), Boston: Kluwer, 2004, pp. 335-352.

Grudin, J. "Desituating Action: Digital Representation of Context," Human-Computer Interaction (16), 2001, pp. 269-286.

Henfridsson, O, and Lindgren, R. "Multi-Contextuality in Ubiquitous Computing: Investigating the Car Case through Action Research," Information and Organization (15:3), 2005.

Hevner, A. R., March, S.T., Park, J., and Ram, S. "Design Science in Information Systems Research," MIS Quarterly (28:1), 2004, pp. 75-105.

Jessup, L. M., and Robey, D. "The Relevance of Social Issues in Ubiquitous Computing Environments," Communications of the ACM (45:12), 2002, pp. 88-91.

Lindgren, R., Henfridsson, O., and Schultze, U. "Design Principles for Competence Management Systems: A Synthesis of an Action Research Study," MIS Quarterly (28:3), 2004, pp. 435472.

Lyytinen, K., and Yoo, Y. "Issues and Challenges in Ubiquitous Computing," Communications of the $A C M(45: 12), 2002 \mathrm{a}$, pp. 63-65.

Lyytinen, K., and Yoo, Y. "Research Commentary: The Next Wave of Nomadic Computing," Information Systems Research (13:4), 2002b, pp. 377-388.

Markus, M. L., Majchrzak, A., and Gasser, L. "A Design Theory for Systems That Support Emergent Knowledge Processes," MIS Quarterly (26:3), 2002, pp. 179-212.

Olsson, C. M. "Exploring the Impact of a Context-Aware Application for In-Car Use," in Proceedings of $25^{\text {th }}$ International Conference on Information Systems, R. Agarwal, L. Kirsch, and J. I. DeGross (Eds.), 2004, pp. 11-21.

Olsson, C. M., and Russo, N. "Applying Adaptive Structuration Theory to the Study of ContextAware Applications," Information Systems Research: Relevant Theory and Informed Practice, B. Kaplan, D. P. Truex III, D. Wastell and A. T. Wood-Harper, and J. I. DeGross (Eds.), Boston: Kluwer, 2004, pr. 735-741.

Orlikowski, W. J. "The Duality of Technology: Rethinking the Concept of Technology in Organizations," Organization Science (3:3), 1992, pp. 398-427.

Patton, M. Q. Qualitative Research and Evaluation Methods $\left(3^{\text {rd }}\right.$ ed.), London: Sage Publications, 2002. 
Robey, D., and Markus, M. L. "Beyond Rigor and Relevance: Producing Consumable Research about Information Systems," Information Resources Management Journal (11:1), 1998, pp. 7-15.

Schmidt, A., Beigl, M., and Gellersen, H.-W. "There is More to Context than Location," Computers and Graphics (23:6), 1999, pp. 893-901.

Susman, G., and Evered, R. "An Assessment of the Scientific Merits of Action Research," Administrative Science Quarterly (23), 1978, pp. 582-603.

Walls, J. H., Widmeyer, G. R., and El Sawy, O. A. "Building an Information Systems Design Theory for Vigilant EIS," Information Systems Research (3:1), 1992, pp 36-59.

Walker, G. H., Stanton, N. A., and Young, M. S. "Where is Computing Driving Cars?," International Journal of Human-Computer Interaction (13:2), 2001, pp. 203-229.

Walsham, G. "Actor-Network Theory and IS Research: Current Status and Future Prospects," in Information Technology and Changes in Organizational Work, W. Orlikowski, G. Walsham, M. R. Jones and J. I. DeGross (Eds.), London: Chapman and Hall, 1996, pp. 466480.

Want, R., Hopper, A., Falcão, V., and Gibbons, J. "The Active Badge Location System," $A C M$ Transactions on Information Systems (10:1), 1992, pp. 91-102.

Want, R., Pering, T., Borriello, G., and Farkas, K. I. "Disappearing Hardware," IEEE Pervasive Computing (1:1), 2002, pp. 36-47.

Weiser, M. "The Computer for the 21 st Century," Scientific American, September 1991, pp. 94104.

\section{ABOUT THE AUTHORS}

Carl Magnus Olsson is a Ph.D. student at the University of Limerick, Ireland. He conducted the research reported here in the Telematics Group of the Viktoria Institute, Sweden, and holds a guest researcher position at the Operations Management and Information Systems Department of Northern Illinois University, USA. He was a participant in the ICIS Doctoral Consortium in Seattle, 2003, and his publications within ubiquitous information environments include conference papers at IFIP WG 8.2, IFIP WG 8.6 and ICIS. Carl can be reached by e-mail at cmo@viktoria.se.

Ola Henfridsson manages the Telematics Group at the Viktoria Institute, Göteborg, Sweden. He is also an assistant professor in Informatics at the School of Information Science, Computer and Electrical Engineering, Halmstad University. Dr. Henfridsson holds a Ph.D. degree in Informatics from Umeå University, Sweden, and is a member of the editorial board of the Scandinavian Journal of Information Systems. Dr. Henfridsson has published his research in journals such as Information Technology and People, Information and Organization, and MIS Quarterly. He can be reached by e-mail at ola@viktoria.se.

\section{APPENDIX}

The game itself can be described as a relatively straightforward shoot-em-up with added tactical elements. The screenshot on the left shows the radar and the road network around the car. A parking space can be seen about $\mathbf{1 5 0}$ meters behind where the car is right now. The scale is shown above the radar ( 500 meters radius is chosen here). By clicking the parking space, the player can initiate missions after reading about them, their level of difficulty, and rewards if completed. 

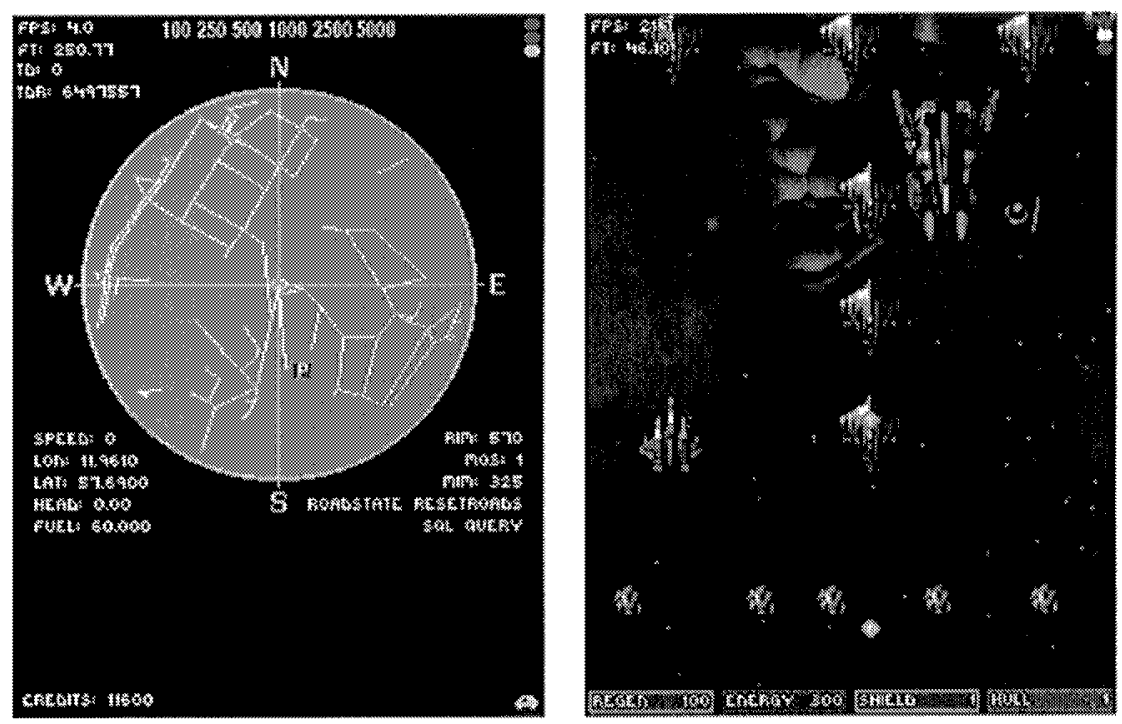

The screenshot on the right is from a mission as it is being played. The mid-left ship is the player, the big space-ship is the driver workload monster indicating an occupied driver, the Tshaped formation of ships are enemy spaceships and the string of round balls are the result of an excessive speeding violation. Fortunately, the driver is currently driving environmentally sound (low fuel consumption) as is indicated by the full regeneration bar in the bottom left corner. This also affects the recuperation of energy spent (second from left, bottom) when the player moves her ship, shoots, and needs to recharge her shield (third from left, bottom) after getting hit by the enemy. The bottom right bar shows how much is left of the hull-once it reaches zero, the mission fails. 